\title{
Les dimensions de la perspective, quand les modes de représentations européennes et chinoises rencontrent l'image numérique
}

\author{
Laurent Lescop ${ }^{1, *}$, et Yang $\mathrm{Lu}^{1,2}$ \\ ${ }^{1}$ AAU-CRENAU, UMR-CNRS 1563, ENSA Nantes, 6 Quai F. Mitterrand, 44262 Nantes, France \\ ${ }^{2}$ Hochschule Anhalt, Seminarplatz 2a Postfach 221506846 Dessau, Allemagne
}

\begin{abstract}
Résumé. Les codes de représentation pour la 3D utilisée pour les visites virtuelles s'appuient sur ceux de la perspective classique telle que décrite par Brunelleschi. Est-il possible dès lors de concevoir une visite virtuelle en utilisant les codes de la perspective chinoise ? La perspective traditionnelle chinoise organise l'espace selon trois valeurs qui organisent les objets dans l'espace et le temps. La compréhension de ces différences doit permettre de construire un espace de représentation immersif dont l'expérience sera fort différente de celles proposées généralement.
\end{abstract}

Mots-clés. Représentation, perspective, immersion, perception, 3D, scénologie, ambiances.

\begin{abstract}
The representation codes used for 3D virtual tours are based on the classical perspective described by Brunelleschi. Is it possible to design a virtual tour by using the codes of the Chinese perspective? The traditional Chinese perspective organises space according to "three distances" to place objects in space and time. The understanding of the "three distances" should allow the building of an immersive represented space in which the experience will be very different from that being usually proposed.
\end{abstract}

Keywords. Representation, perspective, immersion, perception, 3D, scenology, ambiances.

\section{Rendez-vous à Derwentwater}

Dans son célèbre ouvrage L'art et l'illusion, l'historien de l'art Ernst Gombrich (Gombrich, 1971) met en parallèle deux illustrations réalisées l'une par un artiste chinois, Chiang Yee (figure 1), l'autre par un anglais resté anonyme (figure 2). L'aquarelle rehaussée d'encre de Chiang Yee donne à voir un lac avec au premier plan quatre vaches qui paissent à l'ombre de deux arbres noueux. Par-delà l'étendue d'eau, des montagnes émergent de la brume. Le lac n'a que très peu de présence, à peine fait-il quelques clapots.

\footnotetext{
${ }^{*}$ Corresponding author: laurent.lescop@crenau.archi.fr
} 


\section{SCAN'18}

L'anglais compose autour du même thème, il donne cependant plus de précisions, des haies rythment le paysage et induisent des distances, les montagnes se reflètent dans les eaux du lac, une barque semble intriguer les vaches également présentes. Prises indépendamment, ces deux illustrations évoqueraient sans hésitation aucune, l'une un paysage chinois, l'autre un site anglais, il s'agit pourtant du même endroit. Les deux artistes ont représenté une vue de Derwentwater, l'un des lacs de ce comté de Cumbria dans le nord-ouest de l'Angleterre.

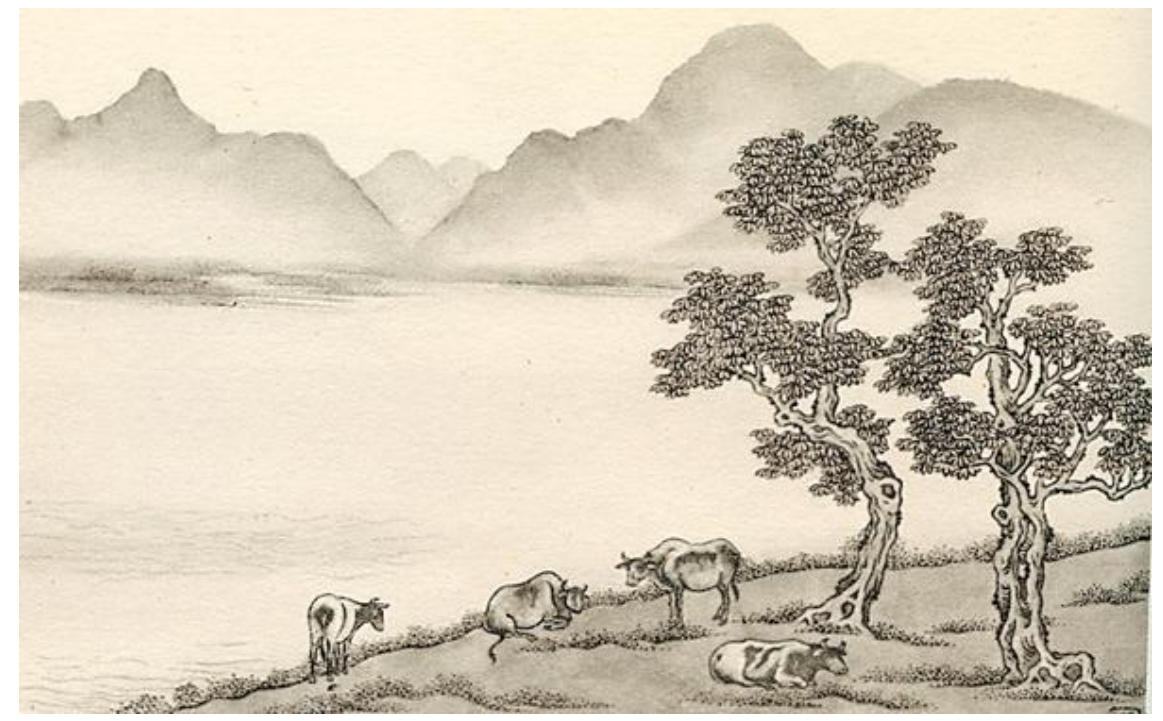

Figure 1. Chiang Yee 'Cows in Derwentwater', 1937, ink on paper, reproduced in 'The Silent Traveller: A Chinese Artist in Lakeland' (1937).

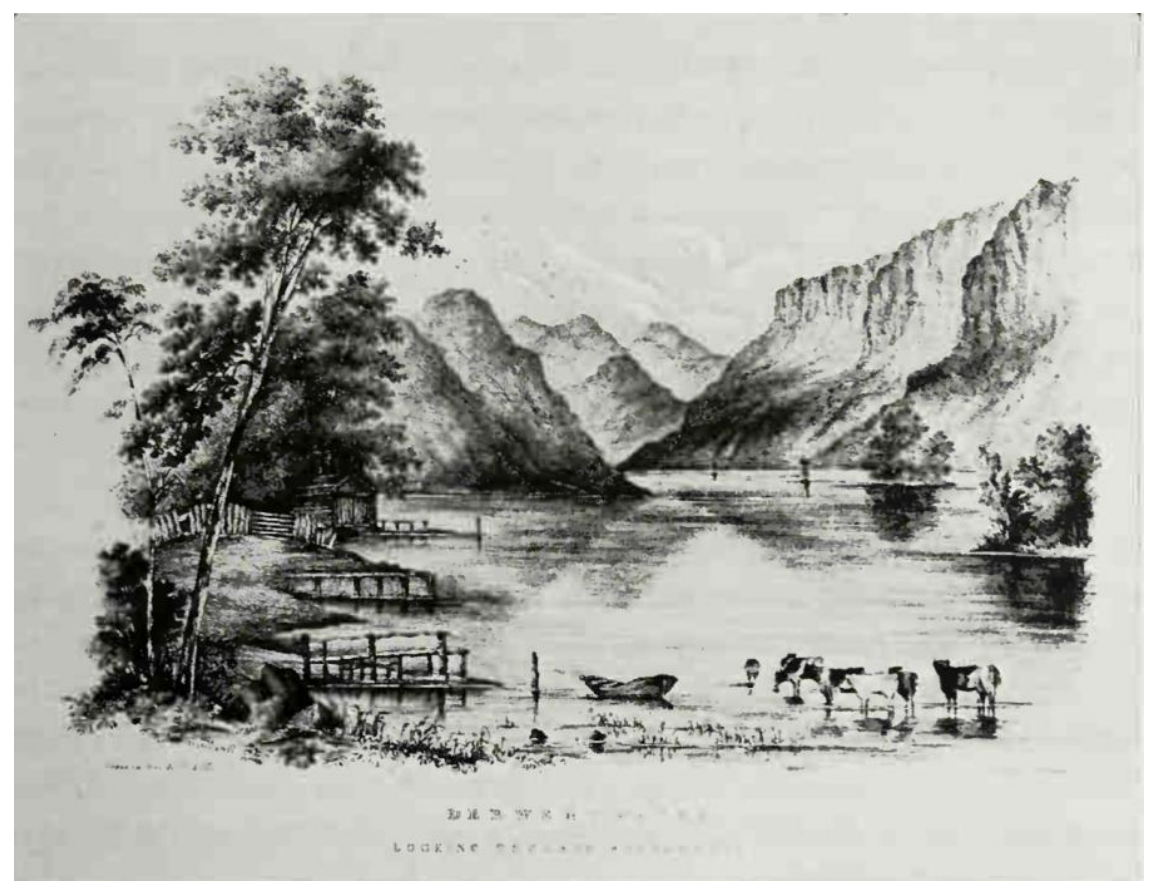

Figure 2. Anonymous, Derwentwater, looking toward Borrowdale, 1826, Lithograph, Crown Copyright Victoria and Albert Museum, London. 
Cette différence d'expression que l'on perçoit parfaitement dans la juxtaposition de ces deux images ne traduit pas seulement ce que Zola appelait déjà « un coin de la création vu à travers un tempérament » (Zola, 1866) selon la formule mainte fois reprise, mais également le déterminisme culturel qui traduit graphiquement une conception de l'espace. La question que nous nous posons ici est de savoir si, après avoir saisi l'armature de ces différences, il est possible de composer des mondes numériques immersifs avec un dialogue culturel tel que celui proposé par Gombrich avec le paysage de Derwentwater.

\section{Mathématiser la ressemblance}

L'obsession du détail, du recueil intégral de chaque brin d'herbe tel que le souhaite le peintre Gerhard Richter, caractérise ce qui est décrit dans le monde occidental comme la ressemblance au sujet représenté. La ressemblance fait référence à la mimesis aristotélicienne que l'on associe à l'imitation de la nature. Le terme mimesis a été traduit à partir de la renaissance italienne par représentation sans pour autant résoudre la question de la relation entre représentant et représenté. Ainsi, l'imitation peut soit capturer tout ce qui caractérise le sujet ou bien se garder une distance qui en permet le contrôle. Au XVIIIe siècle, Diderot défend la distance représentative qui permet d'en contrôler la production, Rousseau récuse la césure de la représentation qu'il considère aliénante (Gilbert, 2013). Jean-Jacques Rousseau, dans la Lettre sur les spectacles (Rousseau, 1758), s'insurgeait contre le clivage de la représentation qui séparait l'activité de l'acteur de la passivité du spectateur y trouvant la métaphore d'une domination politique. Rousseau préférait qu'on se trouve immergé dans une fête ou un carnaval, à la fois spectacle et spectateur. Si le déploiement de ce paradigme dans le domaine de l'esthétique est connu et bien référencé, il doit également être observé dans le domaine de la physique et de la compréhension des phénomènes de la nature. L'imitation de la nature passe par la conception de modèles dont la qualité dénote également la distance qu'ils entretiennent avec ce qu'ils sont censés imiter. Ainsi, la représentation du système stellaire relève pour Galilée d'une substitution d'un visible immédiat par une visibilité calculée. A l'heure de l'image numérique, les questions se posent en des termes exactement identiques, avec des modèles cherchant à imiter totalement un phénomène comme celui de la lumière par exemple, et des modèles proposant ce que l'on pourrait qualifier d'expression métaphorique des phénomènes.

A l'origine de ces questionnements, se trouvent ceux du mécanisme de la vision. Hans Belting (Belting, 2012), rappelle que la culture occidentale associe, culture visuelle et production d'images. Le grec antique Empédocle spéculait au Vème siècle avant notre ère que des rayons lumineux sortaient de nos yeux formant ainsi les objets visibles (Maitte, 2015). Ces rayons ne pouvaient être que rectilignes comme le prouvent les règles de proportionnalité des objets selon la distance. On peut d'ailleurs noter que si la théorie d'Empédocle peut nous sembler amusante, c'est pourtant exactement ce principe qui a été développé pour la simulation de la lumière en informatique par la méthode du lancer de rayon. Cette dernière repose sur le calcul des trajectoires des rayons lumineux à partir de l'œil. Comprendre la vision a donc été à la source de la géométrie euclidienne, puis deux chemins se sont dessinés. D'un côté en Orient, inspirées des sources grecques, les recherches du célèbre savant du Xème-XIème siècle Alhazen, aboutissent sur les fondements théoriques de l'optique et de la lumière tandis qu'en Occident, se fonderont les règles de la perspective. La perspective est en effet, la mathématisation rigoureuse des lois de réduction des objets en fonction de la distance selon des principes progressivement mis au point par Brunelleschi, Alberti et Masaccio à partir du XVème siècle.

Cette construction du monde en est cependant une interprétation et se construit comme une croyance, ce que montre Dominique Raynaud en disséquant scrupuleusement les œuvres et écrits de Brunelleschi, Alberti et Masaccio. Il en arrive à la conclusion qu'il 
existe au-dessus de la composante géométrique de la création de la perspective en occident, la construction d'une croyance, celle «d'une révolution renaissante des méthodes de représentation de l'espace » (Raynaud, 2005). Les œuvres écrites ou peintes de ces trois peintres étudiés ne mobilisent pas assez rigoureusement les principes qui leur sont attribués par les autres les commentant. La technique de la perspective est le fruit d'une évolution alimentée par la mise à disposition de sources indisponibles jusqu'alors, puis par la codification académique. Raynaud conclu dès lors qu'il est «improbable que la perspective soit à l'origine de notre conception de l'espace infini, homogène et isotrope ». De fait, c'est ce modèle occidental académique qui sera ensuite utilisé dans les images numériques cherchant à imiter un réel comme étant considéré le plus proche de la vision naturelle. Les rendus 3D favorisent la perspective linéaire avec le rabattement des points en profondeur sur un plan de projection, ensuite un algorithme calcule ceux qui sont visibles ou masqués.

\section{La perspective chinoise}

En occident, si la représentation de l'espace dans une perspective linéaire peut être caractérisée par le concept abstrait de la description géométrique de l'espace physique, la perspective chinoise est basée sur l'expérience et donc sur la description de l'espace mental de perception. Ce qui différencie fondamentalement les approches occidentales et chinoises du monde est que l'occident se concentre sur les objets, tandis que les chinois décrivent l'espace entre ces objets. Cela permet d'introduire ici la notion de distance. Dans la Chine ancienne, la façon de représenter l'espace tridimensionnel n'a pas de nom précis. On retrouve cependant la dénomination projection parallèle oblique $(\mathrm{Wu}, 2004)$, mais il existe une position plus explicite avec l'utilisation du terme distance dans l'ouvrage Hua Shan Shui Xu (l'Introduction à la peinture de paysage) du peintre théoricien du Vème siècle de notre ère, Zong Bing (宗炳, 375-444). De fait, le terme distance, devient un puissant descripteur qui n'est pas sans rappeler les interrogations de Aristote et Rousseau telles que décrites précédemment. La première distance selon Zong Bing serait celle du point de vue. S'il est à l'intérieur du paysage dans le monde occidental, pour le théoricien chinois la scène paysagère est vue par les yeux et traduite par l'esprit. L'artiste après avoir observé le paysage se retire et va peindre selon sa mémoire, ce n'est donc pas un paysage décrit selon l'observation mais selon la subjectivité du souvenir.

La seconde distance proposée par Zong Bing vient pour la première fois proposer le principe de raccourcissement dans la représentation de la profondeur spatiale dans la peinture de paysage. Cependant, la taille réelle et la taille visuelle des objets n'ont pas de relation proportionnelle stricte, mais reflètent la 《disposition de la nature 》 (自然之势) à travers cette comparaison de tailles. Ainsi, cette représentation bidimensionnelle de l'espace tridimensionnel n'est pas une description objective de l'espace physique, mais plutôt la description de l'expérience subjective de l'espace mental. Guo Si (郭思, fils de Guo Xi) dans son livre Lin Quan Gao Zhi (林泉高致, L'Ambition lointaine dans les forêts et les ruisseaux) a enregistré la pensée de son père Guo Xi (享熙, 1023-1085), qui était artiste et théoricien. On y trouve une définition de ce qui est une structure en trois distances. Guo Xi indique ainsi que : "Une montagne a trois dimensions (distances) : regarder de bas en haut donne la dimension appelée hauteur ; regarder d'avant en arrière donne la dimension appelée profondeur ; regarder en face d'une montagne à partir d'une hauteur opposée donne la dimension horizontale, le niveau. Le ton de la distance de hauteur est clair et lumineux ; celle de la profondeur, lourde et sombre; celle du plan horizontal est quelque chose de clair et parfois sombre. La hauteur est obtenue en exprimant une force ascendante. La profondeur est obtenue en empilant couche après couche. L'effet de la distance est obtenu par l'utilisation de lignes brumeuses qui disparaissent progressivement. 
En peignant des figures humaines dans ces trois dimensions, celles des hauteurs doivent être claires et distinctes; ceux dans les profondeurs détaillées et fines; ceux dans le lointain rêveur et tranquille. Des chiffres clairs et distincts ne devraient pas être courts; ceux qui sont fins et détaillés ne sont pas trop grands; doux et rêveurs pas trop grands. Ce sont les lois des trois dimensions (distance). » (Sakanishi, 1935) (figure 3).
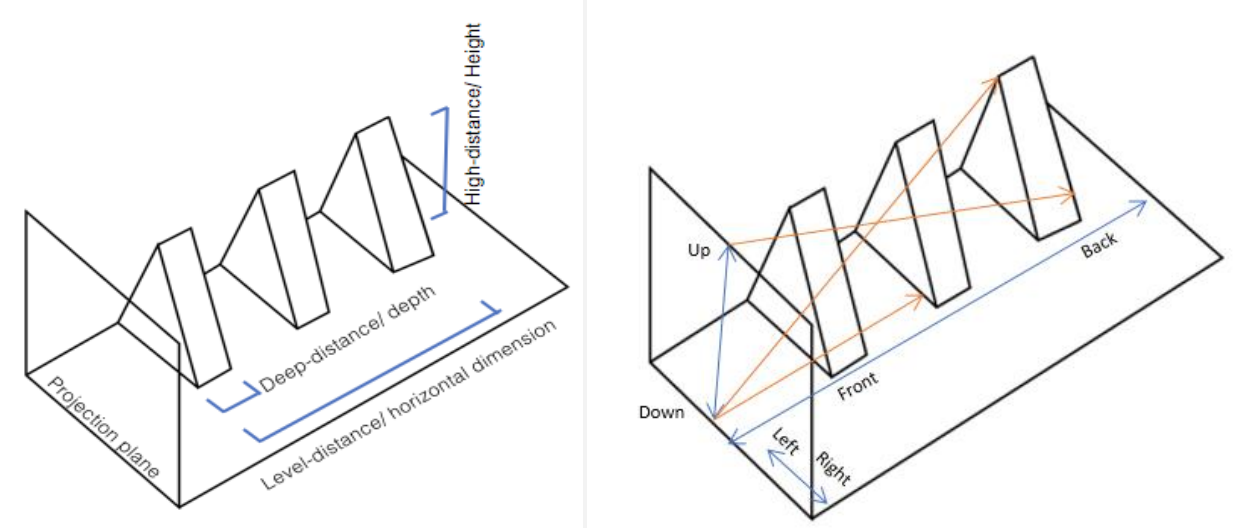

Figure 3. Schématisation des trois distances de Guo Xi dans la peinture du paysage (crédit J. Xiao (2013) et Y. Lu).

Il apparaît donc que les directions des trois distances ne décrivent que deux dimensions de l'espace, haut-bas et avant-arrière, il manque la description d'une troisième dimension latérale gauche-droite. Cela est compensé par une disposition de plans dans la profondeur avec une résolution par chevauchement et occlusion (Fong, 2003). Il n'y a donc pas de continuité entre les motifs. Les transitions entre les plans sont généralement traitées avec un nuage ou du brouillard. Finalement, ce mode de construction correspond à l'expérience du paysage (Feng, 2005): la falaise verticale va susciter un sentiment de sublime et de magnificence, la profondeur exprime le mystère et l'incertitude, l'ampleur renvoie davantage à des ressentis de placidité et d'apaisement. Dans son livre, Shan Shui Chun Quan Ji (山水纯全集, 1121), Han Zhuo (韩拙) complète les trois distances de Guo avec six distances, pour une description plus complète de l'espace. Les trois ajouts sont la distance large (阔远), généralement une étendue d'eau avec une rive dans le premier plan et un balayage spacieux aux montagnes lointaines, ce qui correspond bien au paysage de Derwentwater ; la distance brumeuse (迷远), avec d'épais brouillards qui interrompent les cours d'eau et les plaines et les font disparaître; et enfin la distance obscure (悠远), paysage oblitéré dans l'imprécision et la brume.

Un siècle plus tard, le peintre Huang Gongwang (黄公望, 1269-1354) reprendra la catégorisation en trois distances mais avec les nuances suivantes : «(si) elle continue sans interruption depuis le fond, on l'appelle distance de niveau, quand elle est vue de près par des divisions et des ouvertures alignées, elle est appelée distance éloignée. Une vue lointaine au-delà de la montagne s'appelle haute distance ». (Cahill, 1976). La différence entre Guo et Huang est que pour le premier, les objets sont disposés en fonction d'un point de vue tandis que, pour le second, comme un groupe unitaire cohérent dans lequel il introduit une notion de durée. Gou conçoit le paysage couche par couche, Huang commence lui à accrocher les montagnes aux berges ou les arbres aux collines ce qui induit une continuité de l'espace (figure 4). 

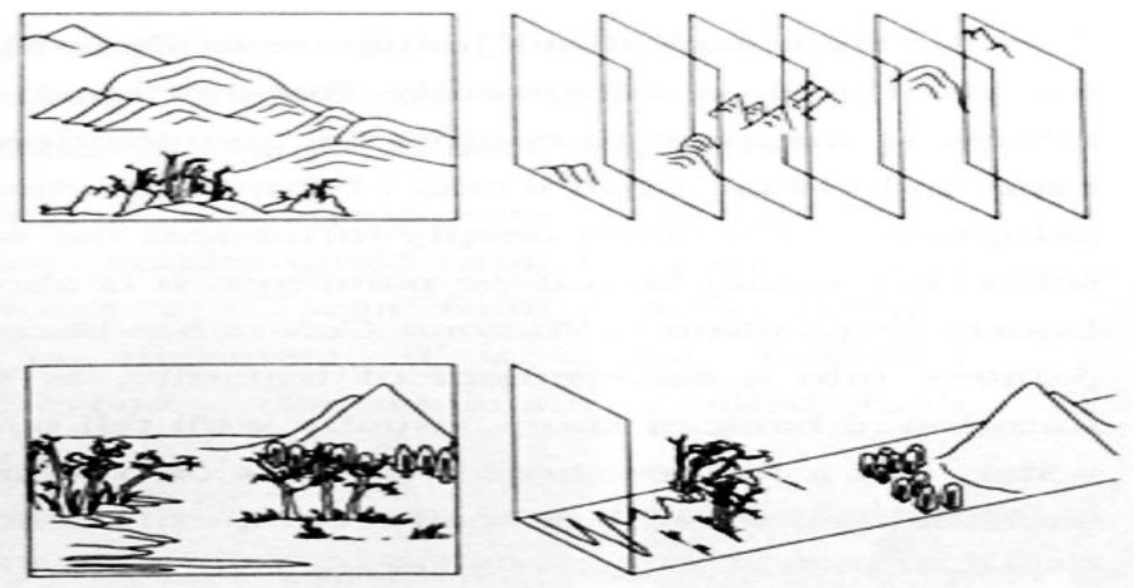

Figure 4. Schématisation des plans séquentiels (crédit WF).

\section{Traductions numériques}

Est-il dès lors possible de traduire, avec les outils numériques dont nous disposons, les dimensions occidentales et chinoises et de produire des images qui traduisent les dimensions de l'univers orthonormé de la peinture européenne et l'organisation des espaces inspirée de Guo ou Huang. Comme pour la construction de toutes images numériques, la première étape est celle de la modélisation où les éléments de l'univers décrit sont sculptés. Puis le rendu passe par une mise en scène de ces éléments en fonction du point de vue. Le passage à une visite immersive induit la possibilité de mettre en mouvement le point de vue et de pouvoir regarder dans toutes les directions (ce que Guo ne pouvait envisager). Le British Museum a promu fin 2017 un film présentant une visite immersive du mont Baiyue (aujourd'hui Qiyun) d'après un rouleau peint en 1623 par Xiang Shengmo. Dans cette animation, l'espace de la montagne est remonté en 3D (figure 5) permettant ainsi une exploration dynamique. De fait, la linéarité de la visite, le continuum spatio-temporel qu'induit la reconstruction proposée traduit bien le cheminement du souvenir mais propose également une trop formelle mensuration en l'espace.

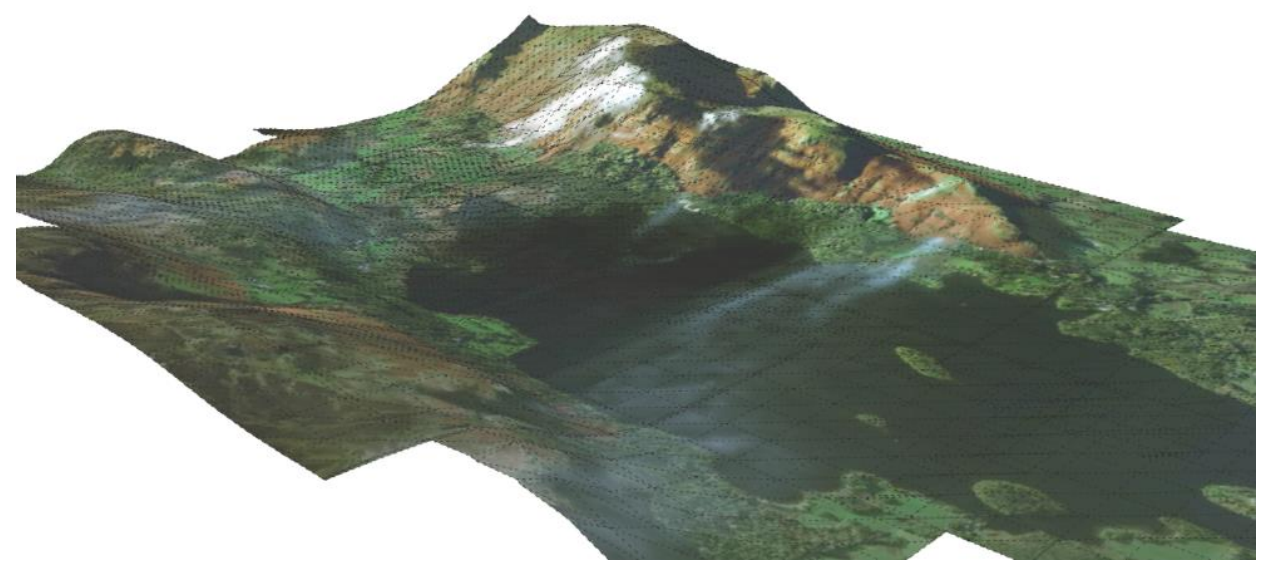

Figure 5. Modélisation du site réel de Derwent Water (crédit L. Lescop). 
Reprenant les vues de Derwent Water, il est faisable de mettre en évidence les deux traitements tridimensionnels préparant la visite virtuelle. Très schématiquement, pour la version occidentale, le paysage est modélisé d'après le point de vue de départ et se présente comme une surface continue épousant les formes et reliefs et parsemée d'objets. Même si la peinture paysagère occidentale a joué des règles de la perspective, il est néanmoins assez aisé de repérer la ligne d'horizon et les lignes de fuites pour la version occidentale du paysage de la figure 2 . En 3D, cela va impliquer la création d'une caméra qui va restituer la position du peintre et l'établissement d'une focale qui déterminera l'effet d'écrasement des plans, la déformation optique des objets et la correspondance du cadre du peintre et celui de la 3D. Il est important de choisir pour ce cadre les mêmes proportions que celles du peintre et de partir sur une focale correspondant plus ou moins à celle de la vision humaine, ce qui, généralement, convient. Si lors d'opérations similaires on trouve du bâti, les outils Camera Match, présents dans de nombreux logiciels 3D, permettent de construire les lignes de fuites à partir de l'image de référence et d'obtenir ainsi automatiquement la ligne d'horizon, la focale et la position de la caméra. Une des applications est la restitution des points de vue à partir d'anciennes photos (Lescop, 2016) (figure 6).
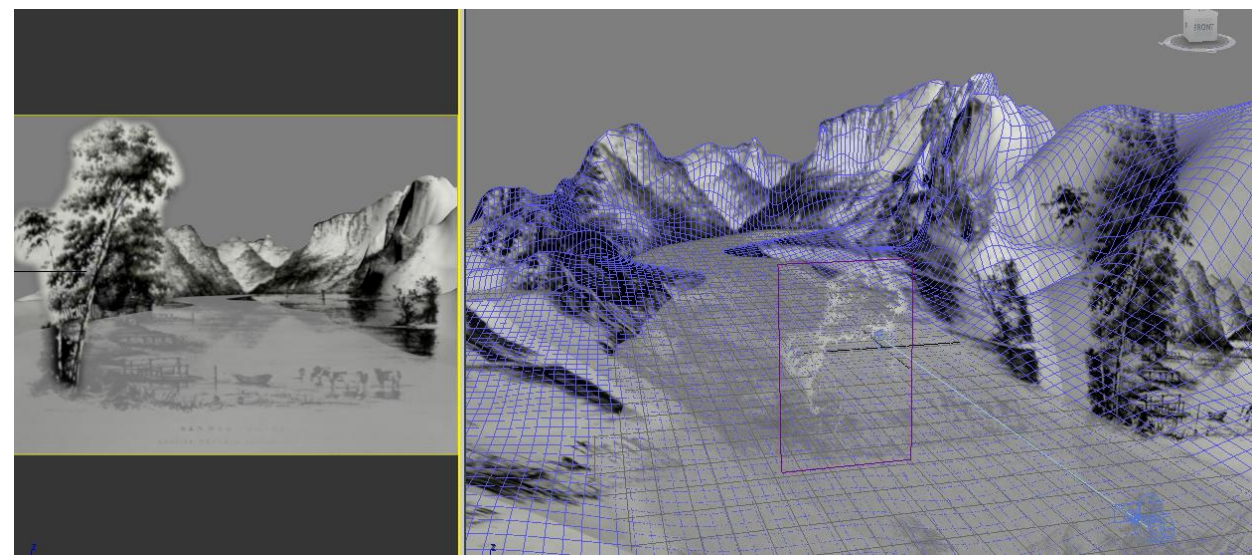

Figure 6. Modélisation du paysage version occidentale (crédit L. Lescop).

Une fois le cadre complet, il reste à compléter ce qui est hors-champ. Pour Derwent Water, nous disposons maintenant des modèles 3D du site, accessibles en ligne via les moteurs de recherche. A vrai dire, la partie la plus complexe consiste à retrouver la position du peintre quelque part autour d'un lac faisant plusieurs kilomètres de long.

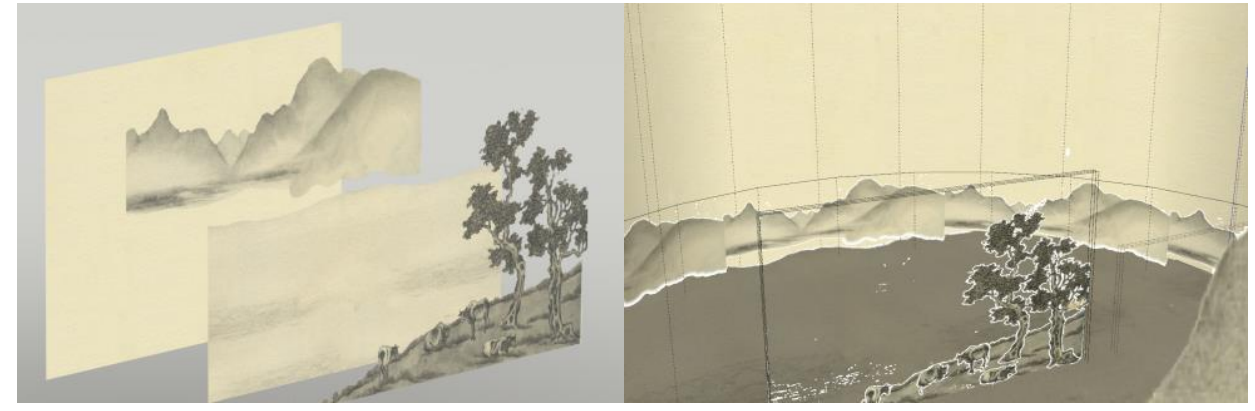

Figure 7. Extrapolation séquentielle du paysage (crédit L. Lescop).

Pour la perspective chinoise, le paysage est saisi et décomposé en plans. La relation spatiale entre chaque plan est l'enjeu principal de la proposition car elle n'est pas 
commensurable mais séquentielle. Si la version occidentale du paysage a réclamé un volume tridimensionnel fait de continuités géométriques d'un plan à l'autre, le paysage «à la chinoise » est, lui, strictement décomposé en plans qui s'organisent dans la hauteur et dans la profondeur. Entre chaque plan, il n'est que du vide. Dans les premières versions, les premières tentatives, une caméra a été posée pour retrouver un point de vue (figures 7 et 8).

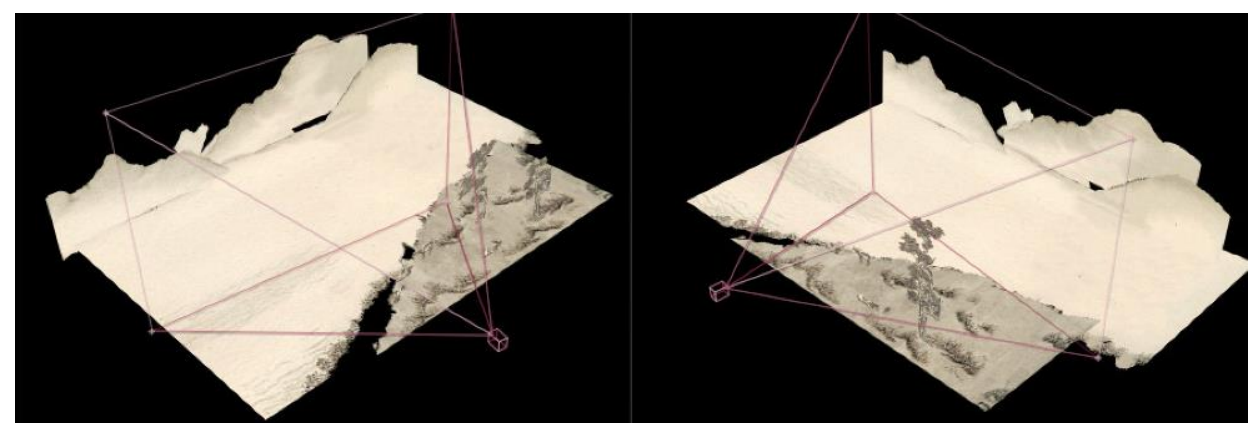

Figure 8. Construction des plans dans After Effect (crédit Y. Lu).

La décomposition en plans n'est pas sans conséquence en termes de perception de l'espace. Si le principe fonctionne bien pour une image fixe ou possédant peu de mouvements, il en est autrement pour des déplacements plus complexes. Au cinéma, la construction des décors en plans successifs associée à la technique du Matte Painting permet de décrire en profondeur des espaces complexes tels que des villes ou de vastes paysages. La technique autorise de petits mouvements de caméra faisant glisser les plans les uns par rapport aux autres sans qu'il ne soit possible de détecter l'astuce (Hamus-Vallée, 2016). Pour des mouvements plus complexes tels que des travellings, il faut procéder autrement. Lors d'un déplacement, les objets proches bougent plus rapidement tandis que les objets lointains restent pratiquement immobiles. Les objets proches sont sensibles aux changements d'échelles par la distance alors que les objets lointains le sont moins. Ainsi, un arbuste à proximité aura une grande dynamique, les montagnes en revanche et plus encore la lune, ne changeront pas de taille ni d'échelle alors que l'on s'en approche. Cette complexité a été très tôt repérée par Walt Disney à qui l'on attribue l'invention de la Caméra Multiplane. Ce dispositif, a en réalité été imaginé par Bill Garity en 1933 pour Disney mais avait déjà été utilisé par Lotte Reiniger pour son film Les aventures du prince Aegmed daté de 1923-1926 (Finch, 1999). Il s'agit de plateaux légèrement espacés recevant les cellulos, chaque plateau pouvant bouger latéralement, indépendamment, simulant ainsi de complexes mouvements de caméra. Le procédé a connu une spectaculaire notoriété grâce au film Blanche Neige réalisé en 1937.

Pour notre exemple, le paysage de Derwent Water se compose finalement de deux plans, celui des arbres et des vaches puis à distance, les montagnes. Entre les deux, le lac produit une mise à distance de la position du peintre à la montagne. La version anglaise contrairement à la version chinoise dispose des objets, arbres, balustrades, arbustes qui conduisent le regard vers la profondeur du tableau donnant par là même une idée précise des échelles de hauteur et de profondeur. Dans l'image de Chiang Yee, le lac impose la distance large (阔远) qui fait que la modélisation des montagnes imposera de les construire en dehors de toutes références géométriques autres que l'impression de hauteur et de distance. De fait, là où la non "géométrification » du paysage va être la plus visible est dans la préservation de la présence visuelle de la montagne avec une valeur identique quel que soit le déplacement. Pour ce faire, au minimum trois solutions : la première consiste à rejeter très loin les massifs et par conséquence à en augmenter considérablement l'échelle afin d'obtenir un effet visuel très éloigné du volume réel. La deuxième solution consiste à 
modifier constamment la focale de la caméra pour compenser la perception d'échelle des montagnes. La troisième solution réside dans l'adaptation de l'échelle des plans contenant les montagnes en fonction de la position de la caméra et de compenser dynamiquement afin qu'elles apparaissent toujours dans les mêmes proportions. Les objets des plans intermédiaires doivent subir les mêmes modifications dans des proportions relatives à leur distance du peintre virtuel. Dans tous les cas, on comprend que ces options ne permettent pas de retrouver les distances en profondeurs et la géométrie de l'espace comme on pourrait le faire à partir d'une perspective à la Brunelleschi.

Le premier plan en revanche peut être une surface modélisée de façon plus classique, mais le lac ne s'exprime plus que par le fait qu'il soutient la brume de laquelle émergent les montagnes. Dans la version anglaise, les eaux réfléchissent les objets environnants, de fines lignes expriment la brise. Dans la version chinoise, le lac met à distance mais n'interagit que très peu avec l'environnement. Il est pure distance (figure 9).

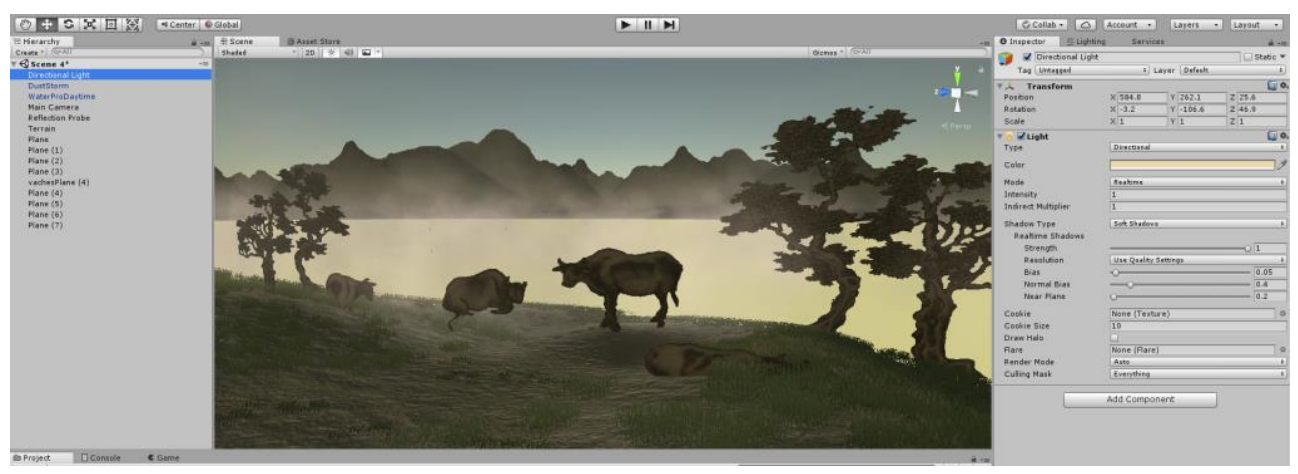

Figure 9. Capture écran de la version immersive chinoise (crédit L. Lescop).

\section{Conclusion}

La transcription numérique de la perspective chinoise demande une approche phénoménologique pour laquelle les outils ne sont pas conçus. Il faut retrouver les notions de dimensions qui traduisent la hauteur, la profondeur, le niveau dans une expérience immersive qui n'est pas sans rappeler ce qui a été conçu pour les panoramas du XIXème siècle avec un proche formé par le faux-terrain et la profondeur par la mise à distance de la toile tandis que la hauteur était donnée par les proportions de celle-là. Un des éléments caractéristiques également de la peinture chinoise est la présence du texte qui décrit et détermine. Cette piste sera à explorer dans la suite des développements en ce qu'elle est distinctive de la peinture occidentale. La position du texte dans un espace immersif est une question à part entière.

Il reste également à définir un protocole de déplacement dans cet espace immersif à la chinoise qui n'est peut-être pas celui du corps, mais celui de l'esprit cheminant en imagination à travers le paysage. Le cadre chinois n'est pas une fenêtre qui capture un point de vue et l'analogie à la fenêtre telle qu'utilisée en occident fonctionne autrement. La fenêtre chinoise ne fait qu'emprunter le paysage, elle ne le capture pas. Cela signifie que le cadre du tableau rassemble les sentiments et non un environnement, ce qui exclut la notion de hors-champ et par là, les tentatives d'immersion en ce qu'elles cherchent à outrepasser le cadre. C'est ce point qu'il s'agit maintenant de résoudre.

Remerciements aux professeurs Johannes Kalvelage, Claus Diessenbacher, Jasper Cepl, HochSchule Anhalt Dessau, Jacques Gilbert, Université de Nantes. 


\section{Bibliographie}

Belting, H. (2012). Contemporary art and the museum in the global age.

Cahill, J F. (1976), Hills Beyond a River: Chinese Painting of the Yuan Dynasty, 1279-1368 (His a History of Later Chinese Painting, 1279-1950; V. 1), WeatherhillClunas, Craig. 1997. Pictures and Visuality in Early Modern China. Reaktion Books.

Feng, W. (2005). An Examination of Chinese Pre-Modern Visual Media, Its Influence on Landscape Ideology, Aesthetics and Relationship to Landscape Experience. University of Sheffield.

Finch C. (1999). L'Art de Walt Disney de Mickey à Mulan, Editions de La Martinière.

Fong, Wen C. (2003). Why Chinese Painting Is History. The Art Bulletin 85 (2), 258-80.

Gilbert J .A. (2013). Les variations de l'imitation, Paris, le Cerf.

Gombrich, E. H. (2000) [1971], Art and Illusion: A Study in the Psychology of Pictorial Representation. Princeton University Press

Hamus-Vallée. R. (2016). Peindre pour le cinéma : Une histoire du matte painting. Presses Universitaires du Septentrion.

Lescop, L. (2016). Ambiance et conception, Livret 2, Mémoire HDR, Université de Nantes. Raynaud, D. (2005). L'émergence de l'espace perspectif: effet de croyance et de connaissance. In A. Berthoz, R. Rech : Les espaces de l'homme, Symposium annuel du Collège de France, 2003, Paris, France. O. Jacob, pp.333-354.

Rousseau, J.-J. (1758). Lettres à d'Alembert sur les spectacles. (Édition Critique). Von Max Fuchs. Lille.

Sakanishi, S. (1935). An Essay on Landscape Painting (林泉高致, Lin Ch'üan Kao Chih written by Guo Xi, translated by Shio Sakanishi). John Murray.

Wu, C. (吴葱). (2004). Beyond Perspectives: Architectural Graphics Study in Culture Perspective (在投影之外: 文化视野下的建筑图学研究). Tianjin University Press (天津大学出版社).

Xiao, J. (2013). Challenging Cavalier Perspective: An Iconological Study of Visual Perception of Depth in Chinese Representational Space. University of Nottingham.

Zola, E. (1866). Mes haines. Collection des Euvres complètes Emile Zola (Paris, n.d), XXIII, 176. 